\title{
New detectors for axions
}

\section{Caterina Braggio*}

INFN and University of Padova

E-mail: caterina.braggio@pd.infn.it

\section{G. Carugno, M. Guarise}

INFN e Università degli Studi di Padova

\section{Santamaria, V. Di Sarno, P. Maddaloni}

CNR-INO

\section{A. Ortolan, G. Ruoso}

LNL, INFN

\section{A. Di Lieto, M.Tonelli, P. Spagnolo}

INFN and University of Pisa

\section{G. Cavoto, J. Ferretti, A. Polosa}

INFN and University of Rome

\section{Capparelli}

UCLA

\section{F. Giazotto}

Scuola Normale Superiore, Pisa

\begin{abstract}
In this work three experimental schemes are described in the field of axion detection that are under investigation within the context of the research call What Next of INFN. AXIOMA is a project based on laser-spectroscopy techniques, QUAX exploits the interaction of the cosmological axion with the spin of fermions (electrons or nucleons) and STAX is an improved detection scheme for a light-shining-through-wall (LSW) experiment with sub-THz photons.
\end{abstract}

INFN Workshop on Future Detectors

16-18 December, 2015

Aula Magna della Cavallerizza Reale, Torino, Italy

\footnotetext{
*Speaker.
} 
In modern cosmology a significant fraction of the universe is matter of unknown form, the so-called dark matter (DM), whose presence is claimed through its gravitational interaction with ordinary barionic matter. Among the DM candidates is the axion, a new particle that followed the introduction of a solution to the strong $\mathrm{CP}$ problem by Peccei and Quinn [1]. A crucial parameter in the Peccei-Quinn theory is the symmetry breaking scale $f_{a}$, and the axion mass is given in terms of $f_{a}$ by

$$
m_{a} \simeq 0.6 \mathrm{eV} \frac{10^{7} \mathrm{GeV}}{f_{a}}
$$

Models with large $f_{a}$, resulting in very weak couplings, establish the axion as the "invisible particle", the most weakly interacting of all particles, if the gravitational force is neglected. An interesting aspect related to driving up the symmetry breaking scale is the consequent cosmological abundance, which makes the axion a favored DM candidate. For certain ranges of $m_{a}$ (from $\mu \mathrm{eV}$ to $\mathrm{meV}$ ), large quantities of axions may in fact have been produced in the early universe that could account for the galactic halo dark matter. It is this type of axion that is addressed in the AXIOMA and QUAX projects, whose detectors are sensitive to an "axion wind" flowing at a velocity of about $10^{-3} \mathrm{c}$ because of the motion of the Solar System through the galactic halo.

Axion-Like-Particles (ALPs) are effective (pseudo)scalar fields, less constrained theoretically, but with interesting implications for dark matter as well. In particular, while for QCD axions the mass, $m_{a}$, and the axion-photon coupling constant, $g_{a \gamma \gamma}$, are directly related, these are independent parameters in the ALPs case. Several experiments have explored the $m_{a} g_{a \gamma \gamma}$ space, but none of them has provided evidence for axion-photon oscillation yet. The present best limit on $g_{a \gamma \gamma}$, although restricted to a narrow window of $m_{a}$ values, was provided by the ADMX Collaboration. The ADMX experiment searches cosmic axions through their conversion into photons in a microwave cavity permeated by a magnetic field. The advantage of laboratory searches, in which the photon (axion) sources are under control, is related to their model-independent incoming axion flux.

Experiments that search for axions from the sun, such as the CERN Axion Solar Telescope (CAST) should also be mentioned [2]. CAST has recently improved the limit on the axion-photon coupling to $g_{a \gamma \gamma}<1.47 \times 10^{-10} \mathrm{GeV}^{-1}$.

In photon regeneration experiments, also called light-shining-through-wall (LSW) experiments, photons from a laser beam may be converted into axions in a strong transverse magnetic field. Unconverted photons get dumped by an opaque obstacle, the "wall", transparent to axions. Behind the obstacle, a second magnetic field may convert axions back into photons, with the same energy as the incoming ones. The regenerated photons are eventually detected and counted. The best laboratory limits on $g_{a \gamma \gamma}$ are set by the OSQAR experiment and by measurements of vacuum magnetic birefringence [3].

STAX is an improved detection scheme for a LSW experiment for axion-like particle searches, as detailed in section 3 .

\section{AXIOMA}

Two novel detection methods based on laser-spectroscopy techniques are investigated in the AXIOMA project, in which the incident particle contributes to the excitation of an atomic or molecular low-lying energy level, as will be detailed in sections 1.1 and 1.2. 


\subsection{Rare-earth doped crystals}

One of the research lines of the AXIOMA project is based on the utilization of wide bandgap materials doped with rare-earth (RE) ions as active detecting material. The requested low detection thresholds are addressed with an all-optical detection scheme based on the infrared quantum counter concept (IRQC), proposed by Bloembergen [12] as a way to extend photon detection to the $1-100 \mu \mathrm{m}$ wavelength range. The incident infrared photon is upconverted in a material that exhibits a four energy level system with $E_{2}>E_{3}>E_{1}>E_{0}$, as those determined in RE-doped materials and kept under the action of a pump laser source resonant with transition $1 \rightarrow 2$ (see Fig. 1 (left)) . In analogy with the infrared quantum counter, detection of the particle is then accomplished
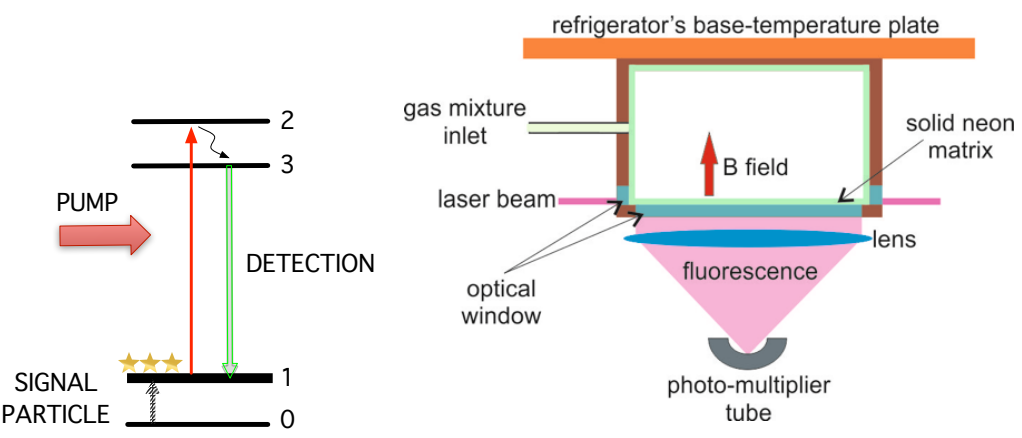

Figure 1: Ideal four-level active material (left). Scheme of the MIS technique (right).

through the fluorescence photons emitted in the transition $3 \rightarrow 1$. The research group has already demonstrated that the IRQC concept can be applied also to the detection of particles [5] and efforts are undergoing to identify a crystal in which the energy threshold is lowered to a few tens of meV.

In this detection method the intrinsic threshold for particle detection coincides with $E_{1}$, which can be as low as a few tens of meV in proper materials, provided the crystal is cooled to a temperature such that the condition $E_{1}<k T$ is satisfied. A cryogenically cooled crystal with $E_{1} \sim 10 \mathrm{meV}$ would then allow extension of the upper limit in axion direct searches. Another essential condition for achieving a detector threshold as low as $E_{1}$ when $E_{1}<k T$ is that the extraction efficiency of the emitted fluorescence photons is highest. Such photons can in fact be lost because of total internal reflection or reabsorption, though the latter can be reduced in rare-earth doped materials by using high purity starting components in an ultra-clean environment.

Interesting crystals under investigation are those in which the fluorescence decays to a metastable level, offering the possibility to exploit looping cycles to increase the detection sensitivity [5].

\subsection{Noble-gas matrices}

The second experimental approach of the AXIOMA project is focused on materials in which a Zeeman splitting is introduced by an external static magnetic field. It has in fact been suggested that dark matter axions may induce atomic transitions between Zeeman states that differ in energy by an amount equal to the axion mass $m_{a}$ [6]. A suitable detection material is then characterized by two magnetic sublevels $a, b$, whose energy difference $E_{b}-E_{a}$ can be tuned via the Zeeman effect to the desired axion mass value. In this approach the range of axion mass that can be probed is 
determined by the atomic/molecular magnetic dipole (M1) transitions, characterized by an energy ranging from $8 \cdot 10^{-5}$ to $2 \cdot 10^{-3} \mathrm{eV}$.

Provided the sample has been cooled in such a way that there are no atoms/molecues in $\mathrm{b}$, as a result of the exposition to the DM axion field $a \rightarrow b$ transitions are expected with a unit-time rate $R_{a b}$ of few events per mole of particles (if M1 selection rules are satisfied) [7]. In order to detect with almost $100 \%$ efficiency the presence of the atoms/molecules promoted into level $b$, a good candidate system is represented by a sample of either some alkali atom or molecular oxygen which is embedded in a condensed phase according to the matrix-isolation spectroscopy (MIS) technique. As shown in Fig. 1 (right), a cryogen-free dilution refrigerator with integrated superconducting magnet provides temperatures as low as $80 \mathrm{mK}$ in magnetic fields up to $18 \mathrm{~T}$. The atomic/molecular species of interest $(\mathrm{S})$ and the noble gas $(\mathrm{N})$ are injected into the MIS cell by capillary filling. In order to perform spectroscopic interrogation of the sample, the MIS cell is equipped with three optical windows: two faced windows permit the propagation of a suitable laser beam; a third window is used to collect the laser-induced fluorescence signal in a perpendicular arrangement. In this configuration, the N-S mixture flowing into the MIS cell sticks to the walls; thus, after some hours of deposition, a noble-gas matrix (incorporating the dopant species $\mathrm{S}$ ) of $1 \mathrm{~mm}$-thickness and several square $\mathrm{cm}$ area is grown on each of the walls. Both Neon-Molecular Oxygen and NeonAtomic Cesium have been analyzed, and an estimate of the the number of axion-induced absorption events is given by expression:

$$
N \cdot N_{A}=\mathscr{R}_{a b} n_{\mathrm{Ne}} V_{c} d(3600 \mathrm{~s}) n_{h}
$$

where reasonable values that might be used are $\mathscr{R}_{a b}=1 \mathrm{~Hz}, n_{\mathrm{Ne}}=4.6 \cdot 10^{22} \mathrm{~cm}^{-3}$ for the neon density, $V_{c} \sim 1 \mathrm{~cm}^{3}$ is the crystal volume, $d=1 \%$ is the doping ratio and $n_{h}=4$ acquisition time in hours. With these numbers $N \cdot N_{A}=10$.

\section{QUAX}

The idea underlying the QUAX (QUaerere AXion) project is inspired by theoretical works $[8,9,10]$, in which the interaction of the cosmological axion with the spin of fermions (electrons or nucleons) is analyzed. The effect of the "axion wind" on a magnetized material is described as an effective oscillating microwave field with frequency determined by $m_{a}$ and amplitude related to $f_{a}$. An axion detector is then implemented through a magnetized sample (see Fig. 2) whose Larmor frequency is tuned to the axion mass by means of an external polarizing static field (e.g. 0.6 T for $17 \mathrm{GHz}$, corresponding to a $70 \mu \mathrm{eV}$ axion mass). The interaction with the axion effective field drives the total magnetization of the sample, whose oscillations can be possibly detected through the LOngitudinal Detection technique (LOD) [11].

It is possible to demonstrate [13] that the equivalent oscillating rf field due to the axion wind has a mean amplitude and central frequency

$$
B_{a}=9.2 \cdot 10^{-23}\left(\frac{m_{a}}{10^{-4} \mathrm{eV}}\right) \mathrm{T}, \frac{\omega_{a}}{2 \pi}=24\left(\frac{m_{a}}{10^{-4} \mathrm{eV}}\right) \mathrm{GHz}
$$

with a relative linewidth $\Delta \omega_{a} / \omega_{a} \simeq 5.2 \times 10^{-7}$. In the LOD technique a pump microwave field $B_{p}$ is applied to the magnetized sample (in a direction orthogonal to the polarizing field) in order 

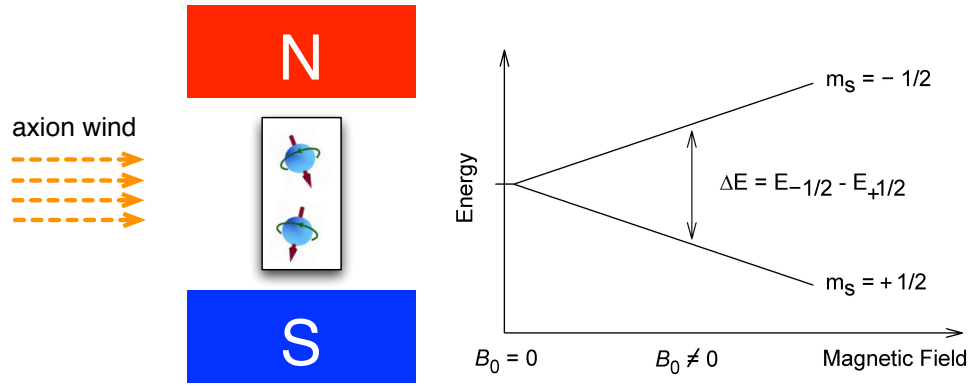

Figure 2: Magnetized material for axion detection in the QUAX experiment.

to amplify the equivalent field generated by the axion wind. The Larmor frequency of electrons is then driven by the rf field

$$
B_{1}=B_{p} \cos \omega_{p} t+B_{a} \cos \omega_{a} t
$$

where $B_{p}>>B_{a}$ is the pump field. Note that the considered axion and pump frequencies $\omega_{a}$ and $\omega_{p}$ lie within the linewidth of the Larmor resonance of the magnetized sample, i.e. $\left(\omega_{p}-\omega_{L}\right)<1 / \tau_{2}^{\star}$ and $\left(\omega_{a}-\omega_{L}\right)<1 / \tau_{2}^{\star}$, with a relaxation time $\tau_{2}^{\star}=1 /\left(\tau_{2}^{-1}+\tau_{r}^{-1}\right)$ and $\tau_{2}$ is the transverse (spinspin) relaxation time and $\tau_{r}$ is the radiation damping characteristic time [12].

Solving the Bloch equations [13], a variable magnetization $\Delta m_{z}$ is produced along the magnetization direction

$$
\Delta m_{z} \equiv M_{z}-M_{0} \simeq \frac{M_{0}}{4} \frac{\tau_{2}^{\star}}{\tau_{2}} \gamma^{2} \tau_{1} \tau_{2}^{\star} B_{p} B_{a} \cos \omega_{D} t
$$

where the detection frequency $\omega_{D}=\omega_{p}-\omega_{a}$ has been defined and with the assumption $\omega_{D}<$ $\min \left\{1 / \tau_{1}, 1 / \tau_{2}^{\star}\right\} . \tau_{1}$ is the longitudinal (spin-lattice) relaxation time. Such low frequency oscillation of the magnetization at the beat frequency between the two rf field components can be detected with suitable devices, e.g. a SQUID amplifier. One advantage of this detection scheme is the possibility to choose $\omega_{D}$ for highest signal to noise ratio. The effective $\mathrm{rf}$ field $B_{a}$ is down converted to a low frequency field with amplitude $B_{D}=\mu_{0} \Delta m_{z} \equiv G_{m} B_{a}$, where $G_{m}=\mu_{0} \frac{M_{0}}{4} \frac{\tau_{2}^{\star}}{\tau_{2}} \gamma^{2} \tau_{1} \tau_{2}^{\star} B_{p}$ is the transduction gain.

If $\tau_{1} \sim \tau_{2}$ and $\tau_{r}<\tau_{2}$, the transduction gain will depend only on the radiation damping time $\tau_{r}$ therefore to obtain $G_{m}>1$ in free space, the sample volume must satisfy the inequality $V_{s}<1 /\left(8 \pi^{2}\right) \lambda_{L}^{3} \tau_{r} \gamma B_{p}$. On the other hand, the pump field amplitude must be far from saturation $\gamma^{2} B_{p}^{2} \tau_{r} \tau_{1}<<1$, which implies $\gamma \tau_{r} B_{p}<<1$. This is the reason why it is not possible to obtain $G_{m}>1$ in free space with realistic sample volumes and pumping fields.

Inside an rf cavity, this problem could be solved but the thermal noise in the hybridized system formed by cavity and magnetized sample is much greater than the axion equivalent field. The solution is to detect axions by placing the sample in a waveguide with a cutoff frequency $\omega_{c}$ above the Larmor frequency of the sample. In these conditions, when the Larmor frequency is lower than $\omega_{c}$, the magnetic resonant mode cannot propagate inside the waveguide and a suppression of the radiation damping mechanism is expected. Another advantage of the detection in the waveguide would be the reduction of the noise associated to thermal photons. 
In this experimental scheme there are at least four sources of noise to consider: a) pump field noise; b) noise at the Axion frequency due to thermal photons that can be down converted after interaction with the pump field; c) magnetization noise generated by sample that can either be down converted by the pump or can act as a noise source in the low frequency readout; d) noise associated with the SQUID pickup at the readout frequency. A preliminary evaluation of these noise sources shows that the most challenging issue is related with the pump field noise, even though any relaxation process that might be active at the readout frequency has to be considered.

\section{STAX}

There are two ways to improve on LSW experiments: by increasing the magnetic fild volume $(L)$ and/or intensity $(H)$ or by increasing the number of photons from the source. With the STAX experiment proposal the second solution is adopted by using gyrotron sources in the far sub- $\mathrm{THz}$ region. In Fig. 3 a sketch of the working region for present and future gyrotrons is reported: more power is attainable decreasing the frequency. A working point above $10 \mathrm{GHz}$ would cut the large

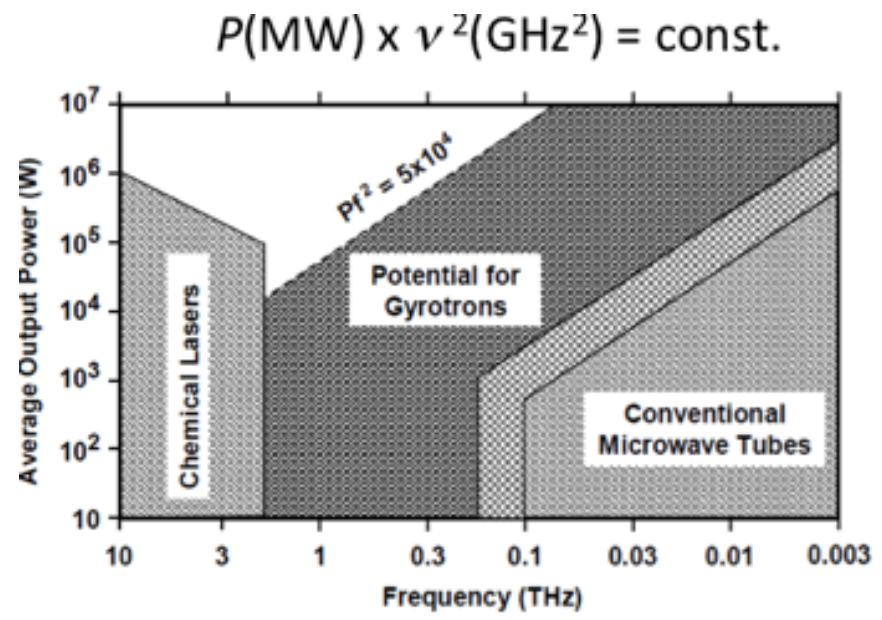

Figure 3: Present and future gyrotrons range.

background on those bands. Gyrotron sources can provide photons from few to several $100 \mathrm{GHz}$. To avoid the ambient microwave background still ensuring a large photon yield, STAX is operated at about $30 \mathrm{GHz}$. In a typical LSW experiment, the rate of events $d N / d t$ is driven by $d N_{\gamma} / d t$, the rate of photons delivered by the source being

$$
\frac{d N}{d t} \sim \frac{d N_{\gamma}}{d t} \cdot P_{\gamma \rightarrow \mathrm{a}} \sim \frac{d N_{\gamma}}{d t} g_{a \gamma \gamma}^{4} H^{4} L^{4}
$$

where $g_{a \gamma \gamma}$ is the (unknown) photon-axion coupling, $H$ is the strength of an external magnetic field and $L$ is the length of the photons path under the action of $H . P_{\gamma \rightarrow \mathrm{a}}=P_{\mathrm{a} \rightarrow \gamma}$ is the probability of photon-to-axion-conversion (and viceversa). If $g_{a \gamma \gamma}<10^{-10} \mathrm{GeV}^{-1}$, as suggested by the CAST

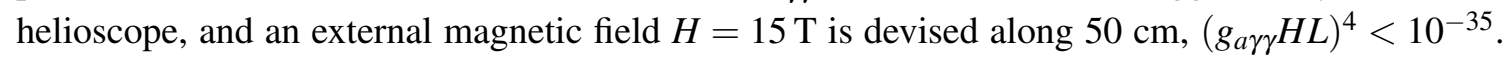
Mega-Watt gyrotron sources can produce $d N_{\gamma} / d t$ over $10^{28}$ photons/second with continuous emission - in the same conditions a $1 \mathrm{~W}$ laser source yielding visible photons can reach $d N_{\gamma} / d t \sim 10^{18}$. 
This would allow $\sim 5 \mathrm{LSW}$ events per year at $g_{a \gamma \gamma} \sim 10^{-10} \mathrm{GeV}^{-1}$. The number of expected events will grow by a factor of $\mathrm{Q}$ if a resonant cavity with quality factor $\mathrm{Q}$ encloses the magnetic field region where photon-to-axion conversion is expected to occur. Detectors sensitive to a single photon in the frequency range around $30 \mathrm{GHz}$ are needed to effectively push below the exclusion limits on $g_{a \gamma \gamma}$. Such devices are not currently available and the proposed LSW set-up needs dedicated research and development on the photon detection side. If detectors of this kind will be engineered, low-energy LSW experiments would be far more reaching at probing low values of $g_{a \gamma \gamma}$ couplings than those based on LASER sources. On the theory side, if the energy of photons from the source is lowered down to the (unknown) mass value of the axion, large numbers of axions at rest might be produced - inactive in a LSW set-up. The computed photon-axion-photon conversion probabilities in this particular limit show that the size $L$ works as an infrared regulator in the conversion probability formula [14].

The proposed configuration can produce an improvement in the exclusion region by at least four orders of magnitude with respect to the ALPS configuration, as proven by explicit calculations. As shown in Fig. 4, this comparison is most effective for axion masses below $0.01 \mathrm{meV}$.
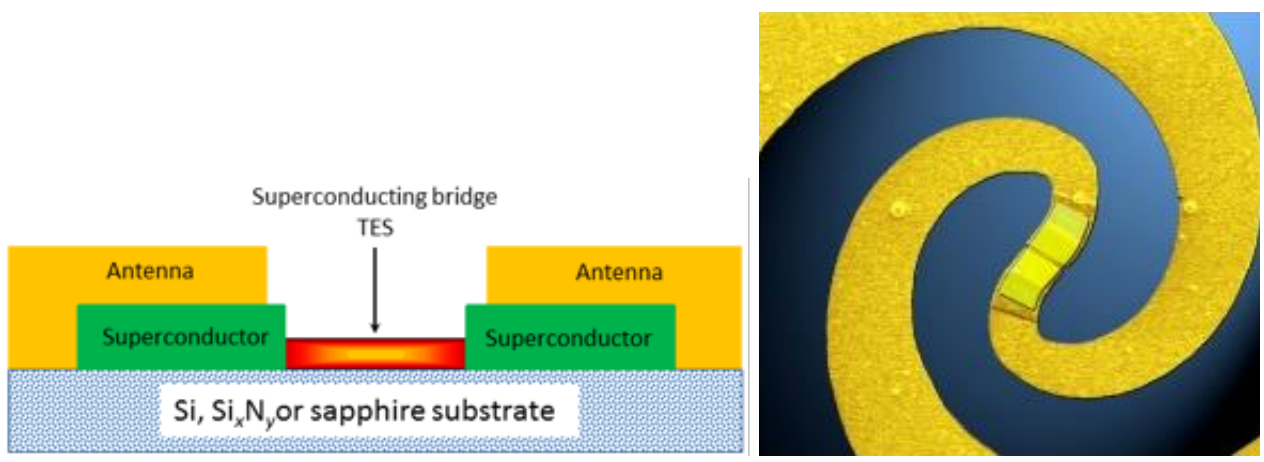

Figure 4: Transition-edge-sensors.

For the development of the photon detector transition-edge-sensors (TES) might be used, operating below $10 \mathrm{mK}$ temperature to detect single photons with an energy of $30-100 \mathrm{GHz}$. The following fundamental issues might be addressed to implement sub-THz single-photon TESs operating in the $30-100 \mathrm{GHz}$ spectral regime:

- choice of a superconductor with a sufficiently low critical temperature $\left(T_{c}<20 \mathrm{mK}\right)$

- tailoring of the TES active volume in order to achieve a reduced thermal capacitance

- design of an integrated highly-efficient planar antenna (a picture of a standard TES (in the center) with the coupled antenna (spiral) is reported below)

- optimization of low-noise SQUID readout electronics

\section{References}

[1] R. Peccei and H. Quinn. Phys. Rev. Lett., 38:1440, 1977. 
[2] M. Arik et al. (CAST Collaboration) Phys. Rev. D, 92:021101(R), 2015.

[3] F. Della Valle, A. Ejlli, U. Gastaldi, G. Messineo, E. Milotti, R. Pengo, G. Ruoso and G. Zavattini. Eur. Phys. J. C, 76: 24, 2016.

[4] N. Bloembergen, Phys. Rev. Lett., 2:84, 1959.

[5] A. F. Borghesani, C. Braggio, G. Carugno, F. Chiossi, A. D. Lieto, M. Guarise, G. Ruoso, and M. Tonelli. Appl. Phys. Lett, 107:193501, 2015.

[6] P. Sikivie. Phys. Rev. Lett., 113:201301, 2014.

[7] L. Santamaria, C. Braggio, G. Carugno, V. D. Sarno, P. Maddaloni, and G. Ruoso. New J. Phys., 17:113025, 2015.

[8] R. Barbieri, M. Cerdonio, G. Fiorentini, and S. Vitale. Phys. Lett. B, 226:357, 1989.

[9] L. M. Krauss, J. Moody, F. Wilczeck, and D. E. Morris. Spin coupled axion detections. Preprint HUTP-85/A006, 1985.

[10] A. I. Kakhizde and I. V.Kolokolov. Sov. Phys. JETP, 72:598, 1991.

[11] J. Hervé and J. C. Pescia. R. Ac. Sci. Paris, 251(665), 1960.

[12] N. Bloembergen and R. V. Pound Phys. Rev., 95(8), 1954.

[13] G. Ruoso, A. Lombardi, A. Ortolan, R. Pengo, C. Braggio, G. Carugno, C. S. Gallo and C. Speake Proceedings of TAUP 2015. Journal of Physics: Conference Series

[14] L. Capparelli, G. Cavoto, J. Ferretti, F. Giazotto, A. Polosa, P. Spagnolo. arXiv1510.06892. 